PROCEEDINGS OF THE

AMERICAN MATHEMATICAL SOCIETY

Volume 131, Number 5, Pages 1591-1600

S 0002-9939(02)06707-2

Article electronically published on September 20, 2002

\title{
ON THE BOCHNER THEOREM ON ORTHOGONAL OPERATORS
}

\author{
ZINOVIY GRINSHPUN
}

(Communicated by Joseph A. Ball)

\begin{abstract}
We prove the following theorem. Any isometric operator $U$, that acts from the Hilbert space $H_{1}(\Omega)$ with nonnegative weight $p(x)$ to the Hilbert space $H_{2}(\Omega)$ with nonnegative weight $q(x)$, allows for the integral representation

$$
\begin{gathered}
U f=\frac{1}{q(\xi)} \frac{\partial^{n}}{\partial \xi_{1} \ldots \partial \xi_{n}} \int_{\Omega} \overline{L(\xi, t)} f(t) p(t) d t \\
U^{-1} f=\frac{1}{p(\xi)} \frac{\partial^{n}}{\partial \xi_{1} \ldots \partial \xi_{n}} \int_{\Omega} \overline{K(\xi, t)} f(t) q(t) d t
\end{gathered}
$$

where the kernels $L(\xi, t)$ and $K(\xi, t)$ satisfy certain conditions that are necessary and sufficient for these kernels to generate the corresponding isometric operators.
\end{abstract}

\section{INTRODUCTION}

The rotation operation in a three-dimensional Euclidean space does not modify the length of vectors, hence it does not modify angles between the vectors. Generalizing this operation to the Hilbert space leads to orthogonal transformations (unitary and isometric operators). By definition, an operator acting on the entire space $H$ is called a unitary operator, if for any two elements in $H$ the transformation preserves their scalar product, hence also the distance between them. A unitary operator is linear, its inverse operator is defined on the entire space and it is a unitary operator that coincides with the adjoint operator. An isometric operator maps one Hilbert space onto another Hilbert space (or its subspace), while preserving the scalar product of the elements. Therefore a unitary operator is a particular case of an isometric operator, which occurs when both spaces coincide. In a finite dimensional space any isometric operator is unitary. This is no longer true in the general case. For example, the operator $U\left(x_{1}, x_{2}, \ldots\right)=\left(0, x_{1}, x_{2}, \ldots\right)$ in a coordinate Hilbert space is isometric but not unitary.

Orthogonal transformations hold a notable place in Operator Theory and its applications.

If $H$ is a separable space, then there exists a closed orthonormal system in $H$. A unitary transformation converts this system into an orthonormal system. Conversely, if there are two closed orthonormal systems and an operator that transforms

Received by the editors April 3, 2001 and, in revised form, January 11, 2002.

2000 Mathematics Subject Classification. Primary 44A05, 44A15, 46F12.

Key words and phrases. Hilbert space, weight function, isometric operator, orthogonal polynomials, Bochner Theorem. 
one of them into another, then this operator is unitary. The well-known integral transforms of Fourier, Plancherel, Watson, Stiltjes, Hilbert and others ([1], 4]) are isometric and, in particular, unitary operators in the corresponding spaces.

The importance of these operators prompted the attention of the renowned mathematician Bochner. The following fundamental theorem belongs to him ([3]):

The Bochner Theorem. For each orthogonal transformation $U$ in the space $L_{2}[a, b]$ there exist two kernels, $K(s, t)$ and $L(s, t)$, that are square integrable with respect to $t$, such that

$$
\begin{gathered}
\int_{a}^{b} K^{2}(s, t) d t=\int_{a}^{b} L^{2}(s, t) d t=s-a \\
\int_{a}^{t} K(s, \tau) d \tau \equiv \int_{a}^{s} L(t, \sigma) d \sigma
\end{gathered}
$$

and for each function $x(t) \in L_{2}(a, b)$

$$
\left.\begin{array}{l}
U x(t)=\frac{\partial}{\partial t} \int_{a}^{b} L(t, \sigma) x(\sigma) d \sigma \\
U^{-1} x(t)=\frac{\partial}{\partial t} \int_{a}^{b} K(t, \tau) x(\tau) d \tau
\end{array}\right\} .
$$

Conversely, two kernels, $K(s, t)$ and $L(s, t)$, that satisfy the conditions $(\alpha)$ and $(\beta)$, define orthogonal transformation in the space $L_{2}(a, b)$ by the formulas $(\gamma)$.

Note that the kernels $K(s, t)$ and $L(s, t)$ are "bad"; at least they cannot be absolutely continuous with respect to $s$ (with square integrable derivative). Otherwise, it would be possible to differentiate under the integral sign and to represent the operator as a Fredholm operator. It is known ([1], [2]) that the integral Fredholm operator $(A \psi)(t)=\int_{a}^{b} K(s, t) x(s) d s$ with the kernel $K(s, t)$, that is square integrable or square integrable with respect to $s$ almost for each $t$, cannot be an orthogonal operator. This is because such an operator is compact and therefore non-invertible, as opposed to an orthogonal operator.

This paper is devoted to the expansion of the Bochner Theorem to isometric operators, acting from the Hilbert space $H_{p(x)}(\Omega)$ to the Hilbert space $H_{q(x)}(\Omega)$, $x \in R^{n}, \Omega=\prod_{i=1}^{n}\left[a_{i}, b_{i}\right]$.

Let $H$ be a set of complex-valued functions $f(x)$ that are defined on the $n$ dimensional block

$$
\Omega=\left\{x=\left(x_{1}, x_{2}, \ldots, x_{n}\right) \in R^{n} \mid a_{i} \leq x_{i} \leq b_{i}, i=\overline{1, n}\right\},
$$

and square integrable on $\Omega$ with the nonnegative weights $p(x)$ and $q(x)$. Consider two Hilbert spaces $H_{1} \equiv H_{p(x)}(\Omega)$ and $H_{2} \equiv H_{q(x)}(\Omega)$, consisting of elements from $H$. The scalar product in the space $H_{1}$ is given by

$$
\left(f_{1}, f_{2}\right)_{p}=\int_{\Omega} p(x) f_{1}(x) \overline{f_{2}(x)} d x
$$

and the scalar product in the space $\mathrm{H}_{2}$ is given by

$$
\left(f_{1}, f_{2}\right)_{q}=\int_{\Omega} q(x) f_{1}(x) \overline{f_{2}(x)} d x .
$$

Let $U$ be an isometric operator from $H_{1}$ to $H_{2}$ that preserves the scalar product. Namely, for every $f_{1}$ and $f_{2} \in H$

$$
\left(f_{1}, f_{2}\right)_{p}=\left(U f_{1}, U f_{2}\right)_{q} .
$$


As is well-known ([1]), an isometric operator is linear, invertible, and the inverse operator is also isometric.

\section{THE MAIN THEOREM}

Theorem 1. For each isometric operator $U$, acting from $H_{1}$ to $H_{2}$, there are two corresponding kernels $K(\xi, x)$ and $L(\xi, x)$, that are defined in $\Omega \times \Omega$ and satisfy the conditions:

$$
\begin{gathered}
\int_{\Omega} K(\xi, x) \overline{K(\xi, x)} q(x) d x=\int_{\Omega_{\xi}} p(x) d x, \\
\int_{\Omega} L(\xi, x) \overline{L(\xi, x)} p(x) d x=\int_{\Omega_{\xi}} q(x) d x, \\
\int_{\Omega_{\xi}} \overline{K(\eta, x)} q(x) d x=\int_{\Omega_{\eta}} \overline{L(\xi, x)} p(x) d x,
\end{gathered}
$$

where $\Omega_{\alpha}=\left\{x \in R^{n} \mid a_{i} \leq x_{i} \leq \alpha_{i}, i=\overline{1, n}\right\} \subseteq \Omega$. The operators $U$ and $U^{-1}$ allow for integral representations for any $f(x) \in H(\Omega)$ :

$$
\begin{gathered}
U f=\frac{1}{q(\xi)} \frac{\partial^{n}}{\partial \xi_{1} \ldots \partial \xi_{n}} \int_{\Omega} f(x) \overline{L(\xi, x)} p(x) d x, \\
U^{-1} f=\frac{1}{p(\xi)} \frac{\partial^{n}}{\partial \xi_{1} \ldots \partial \xi_{n}} \int_{\Omega} f(x) \overline{K(\xi, x)} q(x) d x .
\end{gathered}
$$

Conversely, two kernels, $K(\xi, x)$ and $L(\xi, x)$, that are defined on $\Omega \times \Omega$ and satisfy the conditions (4), (5), and (6), generate the isometric operator $U$ from $\mathrm{H}_{1}$ to $\mathrm{H}_{2}$ and its inverse operator $U^{-1}$, according to formulas (7) and (87).

Proof. We introduce the indicator functions

$$
e_{\xi}(x) \equiv e_{\xi_{1}, \xi_{2}, \ldots, \xi_{n}}\left(x_{1}, x_{2}, \ldots x_{n}\right)= \begin{cases}1 & \text { for } x \in \Omega_{\xi} \\ 0 & \text { for } x \notin \Omega_{\xi}\end{cases}
$$

Set

$$
\begin{gathered}
K(\xi, x)=U e_{\xi}(x), \\
L(\xi, x)=U^{-1} e_{\xi}(x) .
\end{gathered}
$$

Since $U$ and $U^{-1}$ are isometric, we have

$$
\begin{gathered}
\left\|U e_{\xi}(x)\right\|_{q}^{2}=\left\|e_{\xi}(x)\right\|_{p}^{2}, \\
\left\|U^{-1} e_{\xi}(x)\right\|_{p}^{2}=\left\|e_{\xi}(x)\right\|_{q}^{2} .
\end{gathered}
$$

Using the equalities (10) and (11) allows us to rewrite (12) and (13) in the integral form

$$
\begin{aligned}
\int_{\Omega} K(\xi, x) \overline{K(\xi, x)} q(x) d x & =\int_{\Omega_{\xi}} p(x) d x \\
\int_{\Omega} L(\xi, x) \overline{L(\xi, x)} p(x) d x & =\int_{\Omega_{\xi}} q(x) d x .
\end{aligned}
$$

Therefore, we have arrived at equalities (4) and (5). 
For the isometric operator $U$ from $H_{1}$ to $H_{2}$, the following equality holds, $\forall f_{1}, f_{2} \in H$ :

$$
\left(U f_{1}, f_{2}\right)_{q}=\left(f_{1}, U^{-1} f_{2}\right)_{p}
$$

which yields for $f_{1}=e_{\eta}(x), f_{2}=e_{\xi}(x)$

$$
\left(U e_{\eta}, e_{\xi}\right)_{q}=\left(e_{\eta}, U^{-1} e_{\xi}\right)_{p} .
$$

Hence, by taking (10) into consideration, we arrive at the equality (6)

$$
\int_{\Omega_{\xi}} K(\eta, x) q(x) d x=\int_{\Omega_{\eta}} \overline{L(\xi, x)} p(x) d x
$$

for $\forall \xi=\left(\xi_{1}, \xi_{2}, \ldots, \xi_{n}\right), \eta=\left(\eta_{1}, \eta_{2}, \ldots, \eta_{n}\right) \in \Omega$. The equality (14) yields $\forall f(x) \in$ $H(\Omega)$

$$
\left(U f, e_{\xi}\right)_{q}=\left(f, U^{-1} e_{\xi}\right)_{p}
$$

Using the integral form and taking (11) into consideration yields

$$
\int_{\Omega} U f(x) e_{\xi}(x) q(x) d x=\int_{\Omega} f(x) \overline{L(\xi, x)} p(x) d x .
$$

Hence,

$$
\int_{\Omega_{\xi}} U f(x) q(x) d x=\int_{\Omega} f(x) \overline{L(\xi, x)} p(x) d x
$$

Repetitive differentiating of the equality (16) with respect to $\xi_{1}, \xi_{2}, \ldots, \xi_{n}$ yields

$$
\frac{\partial^{n}}{\partial_{\xi_{1}} \partial_{\xi_{2}} \ldots \partial_{\xi_{n}}} \int_{\Omega_{\xi}} U f(x) q(x) d x=\frac{\partial^{n}}{\partial_{\xi_{1}} \partial_{\xi_{2}} \ldots \partial_{\xi_{n}}} \int_{\Omega} f(x) \overline{L(\xi, x)} p(x) d x .
$$

But

$$
\frac{\partial^{n}}{\partial_{\xi_{1}} \partial_{\xi_{2}} \ldots \partial_{\xi_{n}}} \int_{\Omega_{\xi}} q(x) U f(x) d x=q(\xi)(U f)(\xi) .
$$

Therefore

$$
q(\xi)(U f)(\xi)=\frac{\partial^{n}}{\partial_{\xi_{1}} \partial_{\xi_{2}} \ldots \partial_{\xi_{n}}} \int_{\Omega} f(x) \overline{L(\xi, x)} p(x) d x
$$

This implies the representation (7). The equality (14), $\forall f \in H$, implies

$$
\left(U^{-1} f(x), e_{\xi}(x)\right)_{p}=\left(f(x), U e_{\xi}(x)\right)_{q} .
$$


Rewriting this in the integral form and taking (11) into consideration yields

$$
\int_{\Omega_{\xi}} U^{-1} f(x) p(x) d x=\int_{\Omega} f(x) \overline{K(\xi, x)} q(x) d x .
$$

Repetitive differentiating of the equality (20) with respect to $\xi_{1}, \xi_{2}, \ldots, \xi_{n}$ yields

$$
p(\xi) U^{-1} f(x)=\frac{\partial^{n}}{\partial_{\xi_{1}} \partial_{\xi_{2}} \ldots \partial_{\xi_{n}}} \int_{\Omega} f(x) \overline{K(\xi, x)} q(x) d x,
$$

which implies the representation (8).

We now prove the converse statement. Consider the kernels

$$
K(\xi, x) \equiv K\left(\xi_{1}, \xi_{2}, \ldots, \xi_{n}, x_{1}, x_{2}, \ldots, x_{n}\right)
$$

and

$$
L(\xi, x) \equiv L\left(\xi_{1}, \xi_{2}, \ldots, \xi_{n}, x_{1}, x_{2}, \ldots, x_{n}\right)
$$

that are defined on $\Omega \times \Omega$ and satisfy the conditions (4), (5)), and (6). We shall show that the operators $U$ and $U^{-1}$, defined by the formulas (7) and (8), are mutually inverse operators from $\mathrm{H}_{1}$ to $\mathrm{H}_{2}$.

Setting $f(x)=e_{\eta}(x)$ in (7) yields, according to (6),

$$
\begin{array}{r}
U e_{\eta}(x)=\frac{1}{q(\xi)} \frac{\partial^{n}}{\partial_{\xi_{1}} \ldots \partial_{\xi_{n}}} \int_{\Omega} e_{\eta}(x) \overline{L(\xi, x)} p(x) d x \\
=\frac{1}{q(\xi)} \frac{\partial^{n}}{\partial_{\xi_{1}} \ldots \partial_{\xi_{n}}} \int_{\Omega_{\eta}} \overline{L(\xi, x)} p(x) d x \\
=\frac{1}{q(\xi)} \frac{\partial^{n}}{\partial_{\xi_{1}} \ldots \partial_{\xi_{n}}} \int_{\Omega_{\xi}} K(\eta, x) q(x) d x=K(\eta, \xi) .
\end{array}
$$

Setting $f(x)=e_{\eta}(x)$ in (8) yields, according to (6),

$$
\begin{array}{r}
U^{-1} e_{\eta}(x)=\frac{1}{p(\xi)} \frac{\partial^{n}}{\partial_{\xi_{1}} \ldots \partial_{\xi_{n}}} \int_{\Omega} e_{\eta}(x) \overline{K(\xi, x)} q(x) d x \\
=\frac{1}{p(\xi)} \frac{\partial^{n}}{\partial_{\xi_{1}} \ldots \partial_{\xi_{n}}} \int_{\Omega_{\eta}} \overline{K(\xi, x)} q(x) d x \\
=\frac{1}{p(\xi)} \frac{\partial^{n}}{\partial_{\xi_{1}} \ldots \partial_{\xi_{n}}} \int_{\Omega_{\xi}} L(\eta, x) p(x) d x=L(\eta, \xi) .
\end{array}
$$

Condition (4) implies

$$
\left\|U e_{\eta}(x)\right\|_{q}^{2}=\int_{\Omega} K(\eta, x) \overline{K(\eta, x)} q(x) d x=\int_{\Omega_{\eta}} p(x) d x .
$$

Condition (5) implies

$$
\left\|U^{-1} e_{\eta}(x)\right\|_{p}^{2}=\int_{\Omega} L(\eta, x) \overline{L(\eta, x)} p(x) d x=\int_{\Omega_{\eta}} q(x) d x,
$$

but

$$
\left\|e_{\eta}(x)\right\|_{p}^{2}=\int_{\Omega_{\eta}} p(x) d x,\left\|e_{\eta}(x)\right\|_{q}^{2}=\int_{\Omega_{\eta}} q(x) d x .
$$

Therefore,

$$
\left\|U e_{\eta}(x)\right\|_{q}=\left\|e_{\eta}(x)\right\|_{p},\left\|U^{-1} e_{\eta}(x)\right\|_{p}=\left\|e_{\eta}(x)\right\|_{q}
$$


Since linear operators that preserve norms also preserve scalar products, the operators $U$ and $U^{-1}$, defined by (7) and (8), are isometric on the indicator functions for $\forall \xi=\left(\xi_{1}, \xi_{2}, \ldots, \xi_{n}\right) \in \Omega$. Any step function allows for unique representation as a linear combination of the indicator functions $e_{\xi_{1}, \xi_{2}, \ldots, \xi_{n}}\left(x_{1}, x_{2}, \ldots, x_{n}\right) \equiv e_{\xi}(x)$. Therefore the operators $U$ and $U^{-1}$ could be expanded to the step functions while preserving the isometricity property. The set of step functions is dense in $H$. Therefore the operators $U$ and $U^{-1}$ are extendible to the whole space, while preserving isometricity. This concludes the proof of the theorem.

Corollary 1. For $\Omega \equiv[a, b], p\left(x_{1}, x_{2}, \ldots, x_{n}\right)=q\left(x_{1}, x_{2}, \ldots, x_{n}\right) \equiv 1$, we obtain the Bochner Theorem.

\section{Application of the theorem to Trikomi transform}

The Trikomi formulas (see [4]) that relate the standardized Tschebishev polynomials $T_{n}(x)$, orthogonal on $[-1,1]$ with weight $q(x)=\frac{1}{\sqrt{1-x^{2}}}$, with the Legendre polynomials $P_{n}(x)$, orthogonal on $[-1,1]$ with weight $p(x)=1$, have the following representation:

$$
\begin{aligned}
& \left(n+\frac{1}{2}\right)(1+x)^{\frac{1}{2}} \int_{-1}^{x}(x-t)^{-\frac{1}{2}} P_{n}(t) d t=T_{n}(x)+T_{n+1}(x), \\
& \left(n+\frac{1}{2}\right)(1-x)^{\frac{1}{2}} \int_{x}^{1}(t-x)^{-\frac{1}{2}} P_{n}(t) d t=T_{n}(x)-T_{n+1}(x) .
\end{aligned}
$$

Adding the formulas (22) and (23) yields the formula

$$
T_{n}(x)=\frac{2 n+1}{4}\left[\sqrt{1+x} \int_{-1}^{x} \frac{P_{n}(t) d t}{\sqrt{x-t}}+\sqrt{1-x} \int_{x}^{1} \frac{P_{n}(t) d t}{\sqrt{t-x}}\right],
$$

which represents Tschebishev polynomials by means of Legendre polynomials. We transform (24) as follows:

$$
T_{n}(x)=\frac{2 n+1}{4}\left[\int_{-1}^{x} \sqrt{\frac{1+x}{x-t}} P_{n}(t) d t+\int_{x}^{1} \sqrt{\frac{1-x}{t-x}} P_{n}(t) d t\right]
$$

or

$$
T_{n}(x)=\frac{2 n+1}{4} \int_{-1}^{1} G(x, t) P_{n}(t) d t
$$

where

$$
G(x, t)=\left\{\begin{array}{l}
\sqrt{\frac{x+1}{x-t}} \text { for }-1 \leq t<x, \\
\sqrt{\frac{1-x}{t-x}} \text { for } x<t \leq 1 .
\end{array}\right.
$$

The formulas (25) and (26) transform Legendre polynomials into Tschebishev polynomials

$$
\frac{2 n+1}{4} P_{n}(x) \stackrel{U}{\rightarrow} T_{n}(x) .
$$

The operator $U$, acting from $H_{1}[-1,1]$ to $H_{\frac{1}{\sqrt{1-x^{2}}}}[-1,1]$, transforms a complete orthogonal Legendre system into a complete orthogonal Tschebishev system. Therefore $U$ can be extended to the space of functions on $[-1,1]$ that are integrable with 
weight $p(x)=1$ and $q(x)=\frac{1}{\sqrt{1-x^{2}}}$, and $U$ is isometric. We can write it as follows:

$$
U f(x)=\int_{-1}^{1} G(x, t) f(t) d t
$$

It is natural to refer to the operator (27) as the Trikomi operator. According to our theorem, every isometric operator generates two kernels, $K(x, t)$ and $L(x, t)$. We calculate these kernels by following the proof of the theorem.

$$
\begin{gathered}
K(\xi, x)=U e_{\xi}(x), \text { where } e_{\xi}(x)=\left\{\begin{array}{l}
1 \text { for } x<\xi, \\
0 \text { for } x>\xi,
\end{array}-1 \leq x, \xi \leq 1,\right. \\
K(\xi, x)=\int_{-1}^{1} e_{\xi}(t) G(x, t) d t=\int_{-1}^{x} e_{\xi}(t) \sqrt{\frac{x+1}{x-t}} d t+\int_{x}^{1} e_{\xi}(t) \sqrt{\frac{1-x}{t-x}} d t .
\end{gathered}
$$

For $x<\xi$ we have

$$
\begin{array}{r}
K(\xi, x)=\int_{-1}^{x} e_{\xi}(t) \sqrt{\frac{x+1}{x-t}} d t+\int_{x}^{1} e_{\xi}(t) \sqrt{\frac{1-x}{t-x}} d t \\
=\sqrt{x+1} \int_{-1}^{x} \frac{d t}{\sqrt{x-t}}+\sqrt{1-x} \int_{x}^{\xi} \frac{d t}{\sqrt{t-x}} \\
=\left.\sqrt{x+1}(-2 \sqrt{x-t})\right|_{-1} ^{x}+\left.\sqrt{1-x}(2 \sqrt{t-x})\right|_{x} ^{\xi} \\
=2 \sqrt{x+1} \sqrt{x+1}+2 \sqrt{1-x} \sqrt{\xi-x}=2[x+1+\sqrt{(1-x)(\xi-x)}] .
\end{array}
$$

For $x>\xi$ we have

$$
\begin{array}{r}
K(\xi, x)=\int_{-1}^{x} e_{\xi}(t) \sqrt{\frac{x+1}{x-t}} d t+\int_{\xi}^{x} e_{\xi}(t) \sqrt{\frac{x+1}{x-t}} d t+\int_{x}^{1} e_{\xi}(t) \sqrt{\frac{1-x}{t-x}} d t \\
=\int_{-1}^{\xi} \sqrt{\frac{x+1}{x-t}} d t=\sqrt{x+1} \int_{-1}^{\xi} \frac{d t}{\sqrt{x-t}}=\left.\sqrt{x+1}(-2 \sqrt{x-t})\right|_{-1} ^{\xi} \\
=\sqrt{x+1}(2 \sqrt{x+1}-2 \sqrt{x-\xi})=2(x+1-\sqrt{(x+1)(x-\xi)}), \\
\text { since } \int_{\xi}^{x} e_{\xi}(t) \sqrt{\frac{x+1}{x-t}} d t=0 \text { for } \xi<x .
\end{array}
$$

Hence,

$$
K(\xi, x)=\left\{\begin{array}{l}
2[x+1+\sqrt{(1-x)(\xi-x)}] \text { for } x<\xi \\
2[x+1-\sqrt{(x+1)(x-\xi)}] \text { for } x>\xi
\end{array}\right.
$$


According to the theory developed above,

$$
\begin{aligned}
& L(\xi, x)=U^{-1} e_{\xi}(x), \\
& U^{-1} f=\frac{d}{d \xi} \int_{-1}^{1} \frac{f(t) K(\xi, t)}{\sqrt{1-t^{2}}} d t, \\
& \left(U^{-1} f\right)(x)=\frac{d}{d x} \int_{-1}^{1} \frac{K(x, t)}{\sqrt{1-t^{2}}} f(t) d t \\
& =2 \frac{d}{d x}\left[\int_{-1}^{x} \frac{t+1+\sqrt{(1-t)(x-t)}}{\sqrt{1-t^{2}}} f(t) d t\right. \\
& \left.+\int_{x}^{1} \frac{t+1-\sqrt{(t+1)(t-x)}}{\sqrt{1-t^{2}}} f(t) d t\right] \\
& =2 \frac{d}{d x}\left[\int_{-1}^{x} \sqrt{\frac{1+t}{1-t}} f(t) d t+\int_{-1}^{x} \sqrt{\frac{x-t}{1+t}} f(t) d t+\int_{x}^{1} \sqrt{\frac{1+t}{1-t}} f(t) d t\right. \\
& \left.-\int_{x}^{1} \sqrt{\frac{t-x}{1-t}} f(t) d t\right] \\
& =2 \frac{d}{d x}\left[\int_{-1}^{1} \sqrt{\frac{1+t}{1-t}} f(t) d t+\int_{-1}^{x} \sqrt{\frac{x-t}{1+t}} f(t) d t\right. \\
& \left.-\int_{x}^{1} \sqrt{\frac{t-x}{1-t}} f(t) d t\right] \\
& =2\left[\sqrt{\frac{x-x}{1+x}} f(x)+\frac{1}{2} \int_{-1}^{x} \frac{f(t) d t}{\sqrt{(x-t)(1+t)}}\right. \\
& \left.+\sqrt{\frac{x-x}{1-x}} f(x)+\frac{1}{2} \int_{x}^{1} \frac{f(t) d t}{\sqrt{(t-x)(1-t)}}\right] \\
& =\int_{-1}^{x} \frac{f(t) d t}{\sqrt{(x-t)(1+t)}}+\int_{x}^{1} \frac{f(t) d t}{\sqrt{(t-x)(1-t)}} \text {. }
\end{aligned}
$$

Therefore

$$
U^{-1} f(x)=\int_{-1}^{x} \frac{f(t) d t}{\sqrt{(x-t)(1+t)}}+\int_{x}^{1} \frac{f(t) d t}{\sqrt{(t-x)(1-t)}} .
$$

Setting $f(x)=T_{n}(x), U^{-1} f(x)=\frac{2 n+1}{4} P_{n}(x)$ yields

$$
\frac{2 n+1}{4} P_{n}(x)=\int_{-1}^{x} \frac{T_{n}(t) d t}{\sqrt{(x-t)(1+t)}}+\int_{x}^{1} \frac{T_{n}(t) d t}{\sqrt{(t-x)(1-t)}} .
$$

The formula (31) converses (24) and allows us to represent Legendre polynomials by means of Tschebishev polynomials.

We write the formula (30) in a different way:

$$
U^{-1} f(x)=\int_{-1}^{1} f(t) \Phi(x, t) d t, \text { where } \Phi(x, t)=\left\{\begin{array}{l}
\frac{1}{\sqrt{(x-t)(1+t)}} \text { for } t<x \\
\frac{1}{\sqrt{(t-x)(1-t)}} \text { for } t>x .
\end{array}\right.
$$


For $x>\xi$

$$
\begin{array}{r}
L(\xi, x)=U^{-1} e_{\xi}(t)=\int_{-1}^{x} e_{\xi}(t) \frac{d t}{\sqrt{(x-t)(1+t)}}+\int_{x}^{1} e_{\xi}(t) \frac{d t}{\sqrt{(t-x)(1-t)}} \\
=\int_{-1}^{\xi} e_{\xi}(t) \frac{d t}{\sqrt{(x-t)(1+t)}}+\int_{\xi}^{x} e_{\xi}(t) \frac{d t}{\sqrt{(x-t)(1+t)}}+\int_{x}^{1} e_{\xi}(t) \frac{d t}{\sqrt{(t-x)(1-t)}} \\
=\int_{-1}^{\xi} \frac{d t}{\sqrt{(x-t)(1+t)}}=\frac{\pi}{2}-\arcsin \frac{-2 \xi+x-1}{x+1} .
\end{array}
$$

For $x<\xi$

$$
U^{-1} e_{\xi}(t)=\int_{-1}^{x} \frac{d t}{\sqrt{(x-t)(1+t)}}+\int_{x}^{\xi} \frac{d t}{\sqrt{(t-x)(1-t)}}=\frac{\pi}{2}+\arcsin \frac{x-2 \xi+1}{1-x} .
$$

The kernel $L(\xi, x)=U^{-1} e_{\xi}(x)$ can be expressed by the formula

$$
L(x, \xi)=\left\{\begin{array}{l}
\frac{\pi}{2}+\arcsin \frac{x-2 \xi+1}{1-x}, \text { for } x<\xi, \\
\frac{\pi}{2}-\arcsin \frac{x-2 \xi-1}{1+x}, \text { for } x>\xi
\end{array}\right.
$$

According to the theorem, the operator $(U f)(x)$ allows for representation

$$
\begin{aligned}
(U f)(x)= & \sqrt{1-x^{2}} \frac{d}{d x}\left[\int_{-1}^{x}\left(\frac{\pi}{2}-\arcsin \frac{-2 x+t+1}{t-1}\right) f(t) d t\right. \\
& \left.+\int_{x}^{1}\left(\frac{\pi}{2}-\arcsin \frac{-2 x+t-1}{t+1}\right) f(t) d t\right] \\
= & \sqrt{1-x^{2}}\left[\left(\frac{\pi}{2}-\arcsin \frac{-2 x+x+1}{x-1}\right) f(x)+\int_{-1}^{x} \frac{f(t) d t}{\sqrt{(x-1)(t-x)}}\right. \\
& \left.-\left(\frac{\pi}{2}-\arcsin \frac{-2 x+x-1}{x+1}\right) f(x)+\int_{x}^{1} \frac{f(t) d t}{\sqrt{(1+x)(t-x)}}\right] \\
= & \sqrt{1-x^{2}}\left[\left(\frac{\pi}{2}+\arcsin 1\right) f(x)+\int_{-1}^{x} \frac{f(t) d t}{\sqrt{(x-1)(t-x)}}\right. \\
= & \sqrt{1-x^{2}}\left[\int_{-1}^{x} \frac{f(t) d t}{\sqrt{(1-x)(x-t)}}+\int_{x}^{1} \frac{f(t) d t}{\sqrt{(1+x)(t-x)}}\right] \\
= & \sqrt{1-x^{2}}\left[\frac{1}{\sqrt{1-x}} \int_{-1}^{x} \frac{f(t) d t}{\sqrt{x-t}}+\frac{1}{\sqrt{1+x}} \int_{x}^{1} \frac{f(t) d t}{\sqrt{t-x}}\right] \\
= & \sqrt{1+x} \int_{-1}^{x} \frac{f(t) d t}{\sqrt{x-t}}+\sqrt{1-x} \int_{x}^{1} \frac{f(t) d t}{\sqrt{t-x}} .
\end{aligned}
$$

Hence,

$$
(U f)(x)=\sqrt{1+x} \int_{-1}^{x} \frac{f(t) d t}{\sqrt{x-t}}+\sqrt{1-x} \int_{x}^{1} \frac{f(t) d t}{\sqrt{t-x}},
$$

which coincides with the formula (27). The operator (32) is inverse to the Trikomi operator. 


\section{REFERENCES}

[1] Achieser N.J. and Glazman I.M., Theory of Linear Operators in Hilbert Space, Vol. 1, Ungar, New York, 1963. MR 41:9015a

[2] Grinshpun Z.S., Analytic form of isometric operators in weighted Hilbert spaces. Izvestiya Akad. Nauk Kazach. SSR, Ser. Phys.-Mat., 1983, No. 3, pp. 17-20 (Russian). MR 85i:47029

[3] Riesz F. and Nagy B.Sz., Functional Analysis, Ungar, New York, 1955. MR 17:175i

[4] Bateman H. and Erdely A., Higher Transcendental Functions, V. 2, New York, 1953. MR 84h:33001b

Department of Mathematics and Computer Science, Bar-Ilan University, Ramat-Gan 52900, ISRAEL

E-mail address: miriam@macs.biu.ac.il 